\title{
Yager ranking index in fuzzy bilevel optimization
}

\author{
A. Ruziyeva, S. Dempe \\ TU Bergakademie Freiberg, Faculty of Mathematics and Computer Science, Freiberg, Germany \\ Correspondence: A. Ruziyeva. Address: TU Bergakademie Freiberg, Faculty of Mathematics and Computer Science, \\ 09596 Freiberg, Germany. Telephone: 03-731-393-183. Email: ruziyeva@gmail.com.
}

Received: July 2, 2012

Accepted: August 23, $2011 \quad$ Online Published: December 5, 2012

DOI : $10.5430 /$ air.v2n1p55

URL: http://dx.doi.org/10.5430/air.v2n1p55

\section{Abstract}

In the present paper a fuzzy bilevel optimization problem is under discussion. The purpose of the paper consists in finding an optimal solution for this problem. Besides the two well-known approaches (pessimistic and optimistic) there exists a quite new selection function approach, which we present here. A sensible attempt to solve a fuzzy bilevel optimization problem through reformulation to a crisp bilevel optimization problem is considered. Using Yager ranking indices technique an algorithm for searching an optimal solution of the fuzzy bilevel optimization problem is described.

\section{Key words}

Fuzzy bilevel optimization, Bilevel programming, Fuzzy goal programming, Optimal solution

\section{I ntroduction}

Bilevel programming problems are challenging problems of mathematical optimization, which are interesting from the theoretical point-of-view (since it is a special case in nonsmooth optimization) and have a variety of applications. They are hierarchical problems of two decision makers, in which one - the so-called leader - has the first choice and the other one the so-called follower - reacts optimally on the leader's selection. The formulation of the bilevel programming problem for crisp (i.e. with exactly known) data can be found ${ }^{[1]}$. Problems with a predominantly hierarchical structure as a decision-making process are extremely practical to such decentralized systems as agriculture, government policy, economic systems, finance, warfare and are especially suitable for conflict resolutions ${ }^{[1-4]}$.

Considering the inherently difficult nature of bilevel problems due to their nonconvexity, nonsmoothness and implicitly determined feasible set, it is difficult to design convergent algorithms, and the few algorithms that converge appear to be slow most of the time. Even in the simplest case, i.e. when the upper and lower level problems are crisp and linear, the bilevel programming problem has been shown to be NP-hard ${ }^{[5,6]}$.

Moreover, in practical situations data are often not known exactly, i.e. only (subjective) estimates are provided. One commonly used approach to deal with these problems is to model them as fuzzy optimization problems ${ }^{[7]}$. This approach proved very useful in many applied sciences, such as economics, physics ${ }^{[8-11]}$.

As it was shown ${ }^{[12]}$, the Yager ranking indices approach can be very useful in solving (single level) fuzzy optimization problems. In the present paper each fuzzy optimization problem is solved by its reformulation into the crisp optimization 
problem using the Yager ranking indices ${ }^{[13]}$. According to Liu and Kao ${ }^{[12]}$, an optimal solution for a fixed parameter is then taken as an optimal solution of the initial fuzzy optimization problem.

The above concepts are combined, if the data involved in the bilevel optimization problem are known only approximately. A number of fuzzy bilevel programming problems can be found in Dempe et al. ${ }^{[14]}$, Dempe and Starostina ${ }^{[15]}$ and references therein. While some convergent algorithms for crisp bilevel problems exist in the literature (see e.g. Dempe ${ }^{[1]}$, Bard and Moore ${ }^{[16]}$, Ishizuka and Aiyoshi ${ }^{[17]}$ ), solution strategies for fuzzy bilevel programming problems are an emerging new field with a wide range of practical applicability. One of the main problems of a large amount of strategies developed for fuzzy bilevel optimization is a lack of ability to solve bilevel optimization problem. Some of the authors solve this problem as biobjective optimization problems ${ }^{[18]}$. This approach was criticized in Dempe ${ }^{[19]}$ as one, that does not lead to a satisfactory solution.

In the present paper we introduce a sensible attempt to solve fuzzy bilevel optimization problems, that is organized as follows.

Basic notions, such as fuzzy set and fuzzy number, operations on fuzzy numbers, fuzzy order and fuzzy function are presented in Section 2.

A general formulation and our concepts of the bilevel fuzzy optimization are given in Section 3.

The Yager ranking indices technique is formulated for normalized continuous triangular fuzzy numbers in Section 4.

The developed algorithm solves a fuzzy bilevel optimization problem by means of its reformulation to a crisp bilevel optimization problem. For solution of the crisp bilevel optimization problem itself an algorithm is developed. This solution algorithm based on a switch between two (upper and lower) levels is presented in Section 5 for the fuzzy linear bilevel programming problem.

To show that the proposed method is useful in solving real-world problems, the paper is concluded with an illustrative example (the linear toll problem with a fuzzy flow problem on the lower level) in Section 6.

\section{Preliminaries}

In this Section we give such basic definitions as fuzzy set and its level-cut, fuzzy number and its level-cut, fuzzy order, operations with fuzzy (and crisp) numbers and fuzzy function.

\subsection{Fuzzy set and fuzzy number}

Definition 1. A fuzzy set $\tilde{C}$ is defined as a pair $(C, \mu)$, where $C$ is a crisp set $(C \subset \mathrm{R})$ and $\mu_{\tilde{C}}: C \rightarrow[0,1]$ is a membership function of the fuzzy set $\tilde{C}$. For each element $x \in C$, the value of $\mu_{\tilde{C}}(x)$ is called the grade of membership of $x$ in $\tilde{C}$.

Corollary 1. The empty fuzzy set is defined with its membership function $\mu \equiv 0$, i.e. is the same as a crisp empty set.

Definition 2. A fuzzy set $\tilde{C}$ is convex, when for all $x, y \in C$ and for all $\lambda \in[0,1]$ the following inequality holds true:

$$
\mu_{\tilde{C}}(\lambda x+(1-\lambda y)) \geq \min \left\{\mu_{\tilde{C}}(x), \mu_{\tilde{C}}(y)\right\}
$$


A fuzzy number is an extension of a regular number in the sense that it does not refer to one single value but rather to a connected set of possible values, where each possible value has its own "weight" between 0 and 1 . This "weight" is called the membership function. A fuzzy number is thus a special case of a convex fuzzy set. More precisely a fuzzy number was defined by Dubois and Prade ${ }^{[19]}$ as follows.

Definition 3. A real fuzzy number $\tilde{n}$ is a convex continuous fuzzy subset of the real line, whose membership function $\mu_{\tilde{n}}$ is

- A continuous mapping from $R$ to the closed interval [0,1];

- Constant on $(-\infty, c): \mu_{\tilde{n}}(x)=0 \forall x \in(-\infty, c]$;

- $\quad$ Strictly increasing on $[c, a]$;

- Constant on $[a, b]: \mu_{\tilde{n}}(x)=1 \forall x \in[a, b]$;

- $\quad$ Strictly decreasing on $[b, d]$;

- Constant on $(d,+\infty): \mu_{\tilde{n}}(x)=0 \forall x \in[d,+\infty)$.

Here $a, b, c$ and $d$ are real numbers.

Within this Definition we say that $\mu_{\tilde{n}}(x)$ is the truth value of the assertion "the value of $\tilde{n}$ is $x$ ".

The simplest way to define a fuzzy number $\tilde{n}$ is presented by e.g. Buckley ${ }^{[20]}$ as

Definition 4. A continuous triangular fuzzy number $\tilde{n}$ is represented with a triple $\left(n_{L}, n_{T}, n_{R}\right)$, where $n_{L}<n_{T}<n_{R}$ and the membership function $\mu_{\tilde{n}}$ is piecewise-linear.

A possible membership function of triangular fuzzy number $\tilde{n}$ is presented on Figure 1.

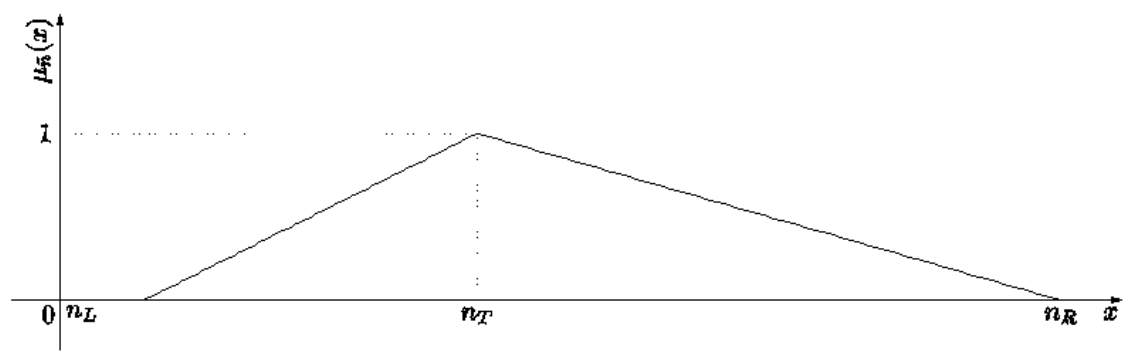

Figure 1. A possible membership function of triangular fuzzy number $\tilde{n}$ 
Definition 5. A level-cut ( $\alpha$-cut, $\alpha$-level) of a fuzzy number $\tilde{C}$ is a special threshold described as an interval $\left[c_{L}(\alpha), c_{R}(\alpha)\right] \subset R$ for some fixed $\alpha \in[0,1]$ (see Figure 5). Here $c_{L}(\alpha)$ and $c_{R}(\alpha)$ represent left- and right-hand side bounds of the fuzzy number $\tilde{c}$ on this certain $\alpha$-cut.

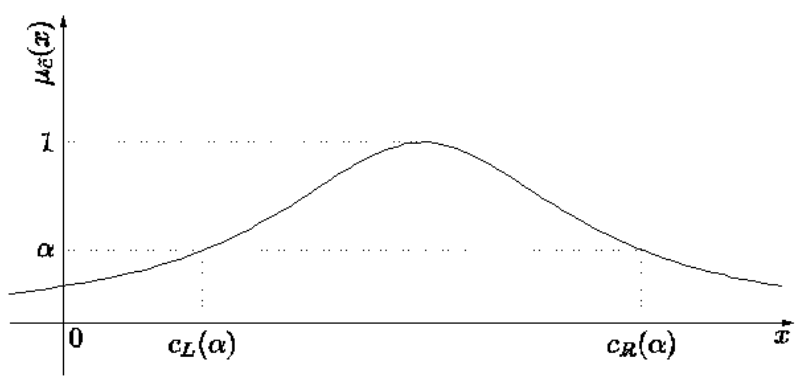

Figure 2. $\alpha$-cut of fuzzy number $\tilde{c}$

Proposition 1. Without loss of generality, from now on we assume that $\tilde{0}=0$, i.e.

$$
\mu_{\tilde{0}}(x)= \begin{cases}1, & x=0 \\ 0, & x \neq 0\end{cases}
$$

Let us extend the concept of a fuzzy set to a space of fuzzy numbers and a fuzzy number to a fuzzy vector.

Definition 6. A fuzzy vector $\tilde{C}$ is an element of the fuzzy space over $R^{n}$ enriched with the nontrivial membership function $\mu_{\tilde{c}}$.

Definition 7. A space of fuzzy numbers $F^{n}$ is defined as a space of all fuzzy vectors.

\subsection{Operations}

Now we give some propositions as respects to the operations on fuzzy vectors. Operations on fuzzy numbers obviously are corollaries.

Proposition 2. Two fuzzy numbers are equal if and only if they have the same membership functions.

Proposition 3. Let $\tilde{a}, \tilde{b} \in F^{n}$. Then the sum of two fuzzy vectors $\tilde{a}+\tilde{b} \in F^{n}$ is defined as

$$
(\tilde{a}+\tilde{b})_{\alpha}=\left[a_{L}(\alpha)+b_{L}(\alpha), a_{R}(\alpha)+b_{R}(\alpha)\right]
$$

where $a_{L}(\alpha)$ and $a_{R}(\alpha)$ are left- and right-side values of the fuzzy vector $\tilde{a}$ on a certain $\alpha$-cut. The same notation is used for the fuzzy vector $\tilde{b}$. 


\subsection{Fuzzy function}

To investigate fuzzy optimization problems we have to define such notions as fuzzy function and its $\alpha$-cut ${ }^{\text {[21] }}$.

Definition 8. A fuzzy function $\tilde{f}$ is an image, such that for every real number / vector $x_{0}$ it sets to conformity a fuzzy number / vector $\tilde{f}\left(x_{0}\right) \in \mathrm{R}^{n}$.

Definition 9. An $\alpha$-cut of a fuzzy function $\tilde{f}(x)$ is defined as an interval $\tilde{f}(x)[\alpha]:=\left[f_{L}(x, \alpha), f_{R}(x, \alpha)\right]$.

An $\alpha$-cut of a fuzzy function $\tilde{f}(x)$ is assumed to be a closed and bounded interval. Thus, $\tilde{f}(x)[\alpha]$ is fully described using the functions $f_{L}(x, \alpha)$ and $f_{R}(x, \alpha)$, which are called the left- and right-hand side functions for the certain $\alpha$ -level of the fuzzy function $\tilde{f}(x)$. Later let us consider the functions $f_{L}(x, \alpha)$ and $f_{R}(x, \alpha)$ depending on $x$ and $\alpha$. Following Panigrahi et al. ${ }^{[22]}$, it is assumed that $f_{L}(x, \alpha)$ is a bounded increasing and $f_{R}(x, \alpha)$ is a bounded decreasing functions of $\alpha$. Moreover, it is obvious that $f_{L}(x, \alpha) \leq f_{R}(x, \alpha)$ for all $\alpha \in[0,1]$.

Definition 10. The fuzzy function $\tilde{f}(x)$ is called convex if for all $\alpha \in[0,1]$ the functions $f_{L}(\cdot, \alpha)$ and $f_{R}(\cdot, \alpha)$ are convex ${ }^{[23]}$.

Continuity and differentiability of the fuzzy function $\tilde{f}(x)$ can also be defined through continuity and differentiability of the left- and right-hand side functions for the fixed aspiration level $\alpha$.

\section{General formulation and basic concepts}

Let the leader select a fuzzy solution $\tilde{c} \in F^{n}$, where $F^{n}$ is the set of fuzzy vectors over $\mathrm{R}^{n}$. Then the follower's task is to solve the problem

$$
\min _{x}\{f(\tilde{c}, x): g(x) \leq 0\}
$$

Where

- $f(\tilde{c}, x): \mathrm{F}^{n} \times \mathrm{R}^{n} \mapsto \mathrm{F}$ is an objective fuzzy function,

- $g(x): \mathrm{R}^{n} \mapsto \mathrm{R}^{p}$ is a constraint crisp function,

- $X(\tilde{c})=\{x: g(x) \leq 0\}$ is a feasible set.

Let $\Psi(\tilde{c})$ denote the set of optimal solutions of fuzzy optimization problem (1), i.e.

$$
\Psi(\tilde{c})=\arg \min _{x}\{f(\tilde{c}, x): g(x) \leq 0\}
$$


Then, the leader's aim is to minimize the upper level fuzzy function $F(\tilde{c}, x)$ subject to $\tilde{c} \in \mathrm{F}^{n}$ and $x \in \Psi(\tilde{c})$. In general, the problem of determining the best solution $\tilde{C}^{*}$ for the leader can be described as that of finding a vector of parameters for fuzzy parametric optimization problem (1), which together with the response to the follower's decision $x(\tilde{c}) \in \Psi(\tilde{c})$ proves to give the best possible value for the upper level objective function $F(\tilde{c}, x)$. That is

$$
\text { "min } "\{F(\tilde{c}, x): x \in \Psi(\tilde{c})\}
$$

Strictly speaking, this definition of the fuzzy bilevel programming problem is valid only in the case of a uniquely determined lower level solution for each possible $\tilde{c}$, i.e. for $|\Psi(\tilde{c})|=1$. The quotation marks in (3) have been used to express this uncertainty in the definition in case of non-uniquely determined solutions.

The assumption that the set of optimal solutions of fuzzy optimization problem (1) reduces to a singleton often is not satisfied ${ }^{[21]}$. That is very common in the fuzzy case of bilevel optimization problems. There exist three possibilities to deal with such kind of problem under assumption that the follower would choose a certain solution.

- The optimistic approach can be used in cooperative case and under assumption that the follower takes the best solution in $\Psi(\tilde{c})$ for the leader.

- The pessimistic approach can be used in non-cooperative case when the leader needs to bound a damage of the follower's choice.

- The follower can select the solution according to a selection function. This approach we call selection function approach and describe it in the present paper.

Let us denote some element of $\Psi(\tilde{C})$ by $x(\tilde{C})$ and assume, that this choice is a fixed selection function for all possible $\tilde{c} \in \mathrm{F}^{n}{ }^{[15]}$. The vector of parameters $\tilde{c}$ describes the "environmental data" for the fuzzy lower level problem (1). The aim of the fuzzy bilevel programming problem is then to select $\tilde{c}$ such that it is an optimal one in this sense:

$$
F(\tilde{c}, x(\tilde{c})) \rightarrow \min _{\tilde{c} \in \mathrm{R}^{n}}
$$

We suggest to compute the selection function $x(\tilde{c})$ using the Yager ranking indices technique. The classical definition in area compensation is defined in Liu and Kao ${ }^{[12]}$ as follows.

Definition 11. For a fuzzy number $\tilde{d} \in C$ the Yager index is computed as

$$
I(\tilde{d})=\frac{1}{2} \int_{0}^{1}\left[d_{L}(\alpha)+d_{R}(\alpha)\right] d \alpha
$$

where $\left[d_{L}(\alpha), d_{R}(\alpha)\right]$ is an $\alpha$-cut of the fuzzy number $\tilde{d}$.

Let us define for a fuzzy vector $\tilde{C}=\left(\tilde{c}_{1} \ldots, \tilde{c}_{n}\right)$ the Yager-index as a vector

$$
I(\tilde{c})=\left(I\left(\tilde{c}_{1}\right), \ldots, I\left(\tilde{C}_{n}\right)\right)
$$


Hence, the function $I(\tilde{C})$ also possesses the properties of linearity and additivity. In the present paper we adopt this method for ranking the objective functions' values (in the lower level).

The aim of the next sections is to compute a selection function $x(\tilde{c}) \in \Psi(\tilde{c})$ using the Yager ranking indices technique on the lower level. Then we find an optimal solution of the fuzzy bilevel optimization problem with the selection function approach.

\section{Fuzzy bilevel optimization problem}

For realizing this idea instead of problem (3) we investigate the fuzzy linear bilevel optimization problem

$$
\begin{gathered}
F(\tilde{c}, x) \rightarrow \min _{\tilde{c} \in \mathrm{R}^{n}} \\
\text { s.t. } x \in \Psi(\tilde{c})
\end{gathered}
$$

with an $n$-dimensional vector of decision variables $x$ under the assumption that the upper level objective function $F(\tilde{c}, x)$ is continuous convex fuzzy function.

Here

$$
\Psi(\tilde{c})=\arg \min _{x}\left\{\tilde{c}^{\mathrm{T}} x: x \in X\right\}
$$

is the set of the optimal solutions on the lower level,

- $X=\{x: A x=b, x \geq 0\}$ is the feasible set,

- $A \in \mathrm{R}^{m \times n}$ is the constraint matrix,

- $\quad b \in \mathrm{R}^{m}$ is the right-hand side vector.

The intention to write (7) means that the lower level fuzzy optimization problem is stated as

$$
\begin{gathered}
\tilde{C}^{\mathrm{T}} x \rightarrow \min \\
x \in X
\end{gathered}
$$

The solution approach to the fuzzy optimization problem supposes to deal with non-comparable nature of fuzzy numbers / vectors $^{[24]}$. Thus, we suggest the following.

According to Liu and Kao ${ }^{[12]}$, as a reformulation of fuzzy optimization problem (8) we obtain the following optimization problem on the lower level:

$$
\begin{gathered}
I(\tilde{c})^{\mathrm{T}} x \rightarrow \min \\
x \in X .
\end{gathered}
$$


Thus, $\Psi_{I}(\tilde{c})=\arg \min _{x}\{I(\tilde{c}) x: x \in X\}$.

Suppose that fuzzy numbers are defined as (normalized) continuous triangular fuzzy numbers $\tilde{C}=\left(c_{L}, C_{T}, C_{R}\right)$, where $c_{L}, C_{T}, C_{R} \in \mathrm{R}^{n}$ (see Definition 4).

Then it is easy to calculate the $\alpha$-cuts:

$$
c_{L}(\alpha)=\left(c_{T}-c_{L}\right) \alpha+c_{L} \text { and } c_{R}(\alpha)=\left(c_{T}-c_{R}\right) \alpha+c_{R}
$$

Using Definition 5 the Yager-index for this particular case is defined as

$$
I(\tilde{c})=\frac{1}{2}\left(c_{T}+\frac{1}{2}\left[c_{L}+c_{R}\right]\right)
$$

This simplifies problem (9) that is already a crisp optimization problem. And now the initial fuzzy bilevel optimization problem (6) is transformed into

$$
\begin{aligned}
& F(I(\tilde{c}), x) \rightarrow \min _{I(\tilde{c}) \in \mathrm{R}^{n}} \\
& \text { s.t.I }(\tilde{c})^{\mathrm{T}} x \rightarrow \min _{x \in X} .
\end{aligned}
$$

\section{Algorithm}

In this Section a solution algorithm for fuzzy bilevel optimization problem (6) is described. We have presented derivations of this problem to crisp bilevel optimization problem (11). To find an optimal solution of problem (11) we suppose to do the following.

STEP 1. For the fixed fuzzy vector $\tilde{c}$ compute the Yager-index $I(\tilde{c})$ and an optimal solution $x^{*}(I(\tilde{c})) \in \Psi(I(\tilde{c}))$ of fuzzy lower level problem (9).

If the optimal solution of lower level problem (9) is not unique, take the best one with respect to the upper level function $F(I(\tilde{c}), x)$.

STEP 2. Fix this solution $x^{*}:=x^{*}(I(\tilde{c}))$ of the follower's problem and solve the upper level problem

$$
\begin{gathered}
F\left(I(\tilde{c}), x^{*}\right) \rightarrow \min _{I(\tilde{c}) \in \mathrm{R}^{n}} \\
\text { s.t. } x^{*}(I(\tilde{c})) \in \Psi(I(\tilde{c})) .
\end{gathered}
$$

STEP 3. Now fix the optimal solution $I^{*}(\tilde{C})$ of problem (12) and repeat Steps 1 to 3 until the solution stops changing. Then the pair $\left(I^{*}(\tilde{C}), x^{*}\right)$ is an optimal solution of bilevel programming problem (11) in sense of Dempe ${ }^{[26]}$. 
STEP 4. Now the inverse function to $I^{*}(\tilde{C})$ has to be found. For the case of triangular fuzzy vectors, according to (10), we choose an optimal $\tilde{C}^{*}=\left(c_{L}^{*}, c_{T}^{*}, c_{R}^{*}\right)$ such that $c_{T}^{*}=I^{*}(\tilde{c}), C_{L}^{*}+c_{R}^{*}=2 I^{*}(\tilde{c})$ and obviously $c_{L}^{*}<c_{T}^{*}<c_{R}^{*}$. With such a triple $\left(c_{L}^{*}, c_{T}^{*}, c_{R}^{*}\right)$ the fuzzy vector $\tilde{C}^{*}$ can be defined by a symmetrical membership function for which $c_{T}^{*}-c_{L}^{*}=c_{R}^{*}-c_{T}^{*}$ holds true. This $\tilde{C}^{*}$ has the Yager-index equal to $I^{*}(\tilde{C})$.

Then the pair $\left(\tilde{C}^{*}, x^{*}\right)$ is an optimal solution of the initial fuzzy bilevel optimization problem (6).

\section{Example}

To accomplish the discussion it is interesting to explain the results by giving a special example - the traffic problem with fuzzy cost coefficients. There the network $G=(V, E)$ consist of a node set $V$ for junctions and an edge set $E$ containing all streets connecting the junctions. Let the upper level objective function $F(c, x)=\left(c^{\text {toll }}\right)^{T} X$ measure the collected money through the traffic network $G$, where $c^{\text {toll }} \in C_{\text {toll }}$ is a vector of the crisp toll charges and $x \in X$ is a traffic flow. Let us define a set $C_{\text {toll }}=\left\{c: c \in Z_{+}^{|T|}, c \leq \bar{c}\right\}$, where $\bar{c}$ is a given upper bound and $T \subset E$ is a set of all toll roads.

Let the lower level objective function $\tilde{f}(c, x)$ measure the quality of the flow $X$, which depends on the toll charges $c^{\text {toll }}$ and the usual user's fuzzy costs such as e.g. fuel $\tilde{c} \in C^{|E|}$, i.e. $c \in \mathrm{R}^{|E|}$. A possible realization could be $\tilde{f}(c, x)=\left(c^{\text {toll }}+\tilde{c}\right)^{T} x$.

Computing the system optimum in the traffic network means that we maximize the collected money for the leader as the upper level objective function value $F(c, x)$ and minimize the total costs for the travel of all users (that we describe as one follower) in the network as the lower level objective function value $\tilde{f}(c, x)$. Clearly these costs do not only depend on the fuzzy costs $\tilde{C}$ for traversing the edges but also on the collected toll charges

$$
c_{p}^{\text {toll }}=\left\{\begin{array}{cc}
c_{p}^{\text {toll }}, & \mathrm{p} \in \mathrm{T} \\
0, & \mathrm{p} \notin \mathrm{T}
\end{array}\right.
$$

where $T \subset E$ is a set of all toll roads. Assume that in the network $G$ there exists at least one toll-free path (i.e. $C_{\text {toll }}$ is bounded).

Using this problem a user equilibrium traffic flow can be computed provided that the costs $\tilde{C}_{e}$ for traversing the edge $e \in E$ are approximately known, i.e. have fuzzy values, and depend on the flow over this edge. Using fuzzy travel costs, the computation of the traffic flow reduces to a fuzzy network flow problem.

Assume that $v$ units of a certain good should be transported with minimal for the users (follower) overall costs from the origin $s \in V$ to the destination $d \in V$ through the traffic network $G$. The problem is to compute optimal amounts of transported goods on the streets of the network. 
Let $x_{e}=x_{k l}$ denote the amount of transported units over the edge $e=(k, l) \in E$, that connects two vertices $k$ and $l$ ( $k, l \in V)$. Let $O_{k}\left(I_{k}\right)$ denote the set of all edges leaving (entering) the node $k$.

Assume that the streets may have different capacities and let the flow $x_{e}$ on the edge $e$ be bounded by the capacity $u_{e}$. This is expressed in the inequality (15) given below. The constraint (16) is used to guarantee that the total incoming flow is equal to the total outgoing flow. Moreover, the outgoing flow at the origin equals to $v$ (see (17)).

$$
\begin{gathered}
F(c, x)=\sum_{e \in T} c_{e}^{\text {toll }} x_{e} \rightarrow \max _{c^{\text {toll }} \in C_{\text {toll }}} \\
\text { s.t. } \tilde{f}(c, x)=\sum_{e \in E} \tilde{c}_{e} x_{e}+\sum_{e \in T} c_{e}^{\text {toll }} x_{e} \rightarrow \min _{x} \\
x_{e} \leq u_{e} \quad \forall e \in E \\
\sum_{k \in I_{l}} x_{k l}-\sum_{i \in O_{l}} X_{l i}=0, \forall l \in V \backslash\{s, d\} \\
\sum_{k \in I_{s}} X_{k s}-\sum_{i \in O_{s}} X_{s i}=-v \\
x_{e} \geq 0
\end{gathered}
$$

Problem (13)-(18) poses in a form of (8) and $\Psi(\tilde{c})$ is the optimal solution set of problem (14)-(18). (Familiar formulation for the lower level problem in a crisp case can be found in Dempe and Lohse ${ }^{[25]}$ ).

Description of a numerical example is the following (for $s=1$ and $d=4$ ):

$$
\begin{aligned}
& F(c, x)=c_{13}^{\text {toll }} x_{13}+c_{23}^{\text {toll }} x_{23}+c_{34}^{\text {toll }} x_{34} \rightarrow \max _{C_{\text {toll }}=[0,5]^{3}} \\
& \text { s.t. } \tilde{f}(c, x)=\tilde{3} x_{12}+\left(\tilde{3}+c_{13}^{\text {toll }}\right) x_{13}+\left(\tilde{3}+c_{23}^{\text {toll }}\right) x_{23}+\tilde{7} x_{24}+\left(\tilde{3}+c_{34}^{\text {toll }}\right) x_{34} \rightarrow \min _{x} \\
& x_{12}+x_{13}=90 \quad 0 \leq x_{12} \leq 90 \\
& x_{24}+x_{34}=90 \quad 0 \leq x_{13} \leq 90 \\
& \text { with demand and capacity } 0 \leq x_{23} \leq 60 \\
& x_{12}=x_{23}+x_{24} \quad 0 \leq x_{24} \leq 30 \\
& x_{13}+x_{23}=x_{34} \quad 0 \leq x_{34} \leq 90
\end{aligned}
$$

The corresponding traffic network is illustrated in Figure 3. 


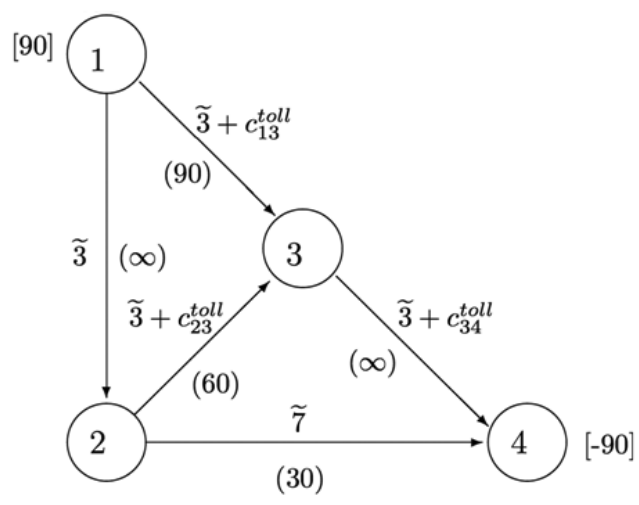

Figure 3. The example of the traffic network

Let fuzzy numbers be defined as continuous triangular fuzzy numbers: $\tilde{3}=(2,3,5)$ and $\tilde{7}=(5,7,8)$. These fuzzy numbers could be described through the corresponding left- and right-hand side function on $\alpha$, i.e. $\tilde{3}$ is described as a pair of functions $\alpha+2$ and $-2 \alpha+5$ for $\alpha \in[0,1]$. Analogously, $\tilde{7}$ is presented by $2 \alpha+5$ and $-\alpha+8$ for $\alpha \in[0,1]$.

Thus, it makes possible to compute Yager index for our future derivations without any troubles. Using (5) we compute Yager-indices:

$$
I(\tilde{3})=3,25 \quad I(\tilde{7})=7,25 .
$$

Now let us implement the above described algorithm for this example.

STEP 1 (Iteration 1). Fix the vector of toll parameters $c^{0}:=\left(c_{13}^{0}, c_{23}^{0}, c_{34}^{0}\right)=(0,0,0)$ and compute an optimal solution of the lower level problem with the following objective function

$$
\tilde{f}\left(c^{0}, x\right)=\tilde{3} x_{12}+\tilde{3} x_{13}+\tilde{3} x_{23}+\tilde{7} x_{24}+\tilde{3} x_{34}
$$

Using results from (19) fuzzy lower level problem (20) can be stated as a crisp linear optimization problem

$$
f\left(c^{0}, x\right)=3,25 x_{12}+3,25 x_{13}+3,25 x_{23}+7,25 x_{24}+3,25 x_{34}
$$

with the same demands and constraints. This problem has as an optimal solution a vector $x_{1}^{*}=(0,90,0,0,90)$.

STEP 2. Now with the fixed solution $x_{1}^{*}$ of lower level problem (21) we solve the following upper level problem:

$$
\begin{gathered}
F\left(c, x_{1}^{*}\right)=c_{13}^{\text {toll }} 90+c_{23}^{\text {toll }} 0+c_{34}^{\text {toll }} 90 \rightarrow \max \\
c \in[0,5]
\end{gathered}
$$

where $c=\left(=c_{13}^{\text {toll }}, c_{23}^{\text {toll }}, c_{34}^{\text {toll }}\right)$ and an optimal solution is $c^{1}=\left(c_{13}^{1}, c_{23}^{1}, c_{34}^{1}\right)=(5,0,5)\left(c_{23}^{1}:=c_{23}^{0}\right)$.

STEP 3. Fix $c^{1}=(5,0,5)$ and go to STEP 1 . 
STEP 1 (Iteration 2). Now we solve initial lower level problem with the fixed vector $c^{1}$ :

$$
\tilde{f}\left(c^{1}, x\right)=\tilde{3} x_{12}+\tilde{8} x_{13}+\tilde{3} x_{23}+\tilde{7} x_{24}+\tilde{8} x_{34}
$$

Where $\tilde{8}=(7,8,10)$ and $I(\tilde{8})=8,25$.

Thus, we obtain the following crisp optimization problem:

$$
f\left(c^{1}, x\right)=3,25 x_{12}+8,25 x_{13}+3,25 x_{23}+7,25 x_{24}+8 x_{34} .
$$

An optimal solution of problem (24) is $x_{2}^{*}=(90,0,60,30,60)$.

STEP 2. With the fixed solution $x_{2}^{*}$ on lower level we solve the following upper level problem:

$$
\begin{gathered}
F\left(c, x_{2}^{*}\right)=c_{13}^{\text {toll }} 0+c_{23}^{\text {toll }} 60+c_{34}^{\text {toll }} 60 \rightarrow \max \\
c \in[0,5]
\end{gathered}
$$

where an optimal solution is $c^{2}=\left(c_{13}^{2}, c_{23}^{2}, c_{34}^{2}\right)=(5,5,5)\left(c_{13}^{2}:=c_{13}^{1}\right)$.

STEP 3. Fix $c^{2}=(5,5,5)$ and go to STEP 1 .

STEP 1 (Iteration 3). With the fixed vector $c^{2}$ the initial lower level problem has to be solved

$$
\tilde{f}\left(c^{2}, x\right)=\tilde{3} x_{12}+\tilde{8} x_{13}+\tilde{8} x_{23}+\tilde{7} x_{24}+\tilde{8} x_{34}
$$

Applying Yager ranking indexes technique to fuzzy optimization problem (26), we obtain the following problem:

$$
f\left(c^{2}, x\right)=3,25 x_{12}+8,25 x_{13}+8,25 x_{23}+7,25 x_{24}+8 x_{34} .
$$

An optimal solution of this problem is $x_{3}^{*}=(30,60,0,30,60)$.

STEP 2. With the fixed solution $x_{3}^{*}$ on lower level the following upper level problem has to be solved

$$
\begin{gathered}
F\left(c, x_{3}^{*}\right)=c_{13}^{\text {toll }} 60+c_{23}^{\text {toll }} 0+c_{34}^{\text {toll }} 60 \rightarrow \max \\
c \in[0,5]
\end{gathered}
$$

where an optimal solution is $c^{3}=\left(c_{13}^{3}, c_{23}^{3}, c_{34}^{3}\right)=(5,5,5)\left(c_{13}^{2}:=c_{13}^{1}\right)$.

It is easy to see that as soon as an optimal solution $x^{*}$ of the lower level optimization problem remains to be optimal, the pair $\left(c^{3}, x_{3}^{*}\right)$ is an optimal solution of the initial fuzzy bilevel programming problem in sense of Dempe ${ }^{[26]}$. 


\section{Conclusions}

A first attempt for developing an algorithm solving the fuzzy bilevel optimization problem is described in the present paper. At the lower level the fuzzy linear optimization problem is solved by the Yager-index ranking technique. This solution is taken to find with a selection function approach an optimal solution on the upper level. This method is illustrated on the example of the traffic problem with given fuzzy costs.

Obviously, the method can be extended to the nonlinear but convex and continuous fuzzy optimization problems on the lower level.

\section{References}

[1] Dempe S., Foundations of Bilevel Programming, Kluwer Academie Publishers, Dordrecht, 2002.

[2] Candler W., Fortuny-Amat J., McCarl B., The potential role of multilevel programming in agricultural economics, American Journal of Agricultural Economics. 1981; 63: 521-531. http://dx.doi.org/10.2307/1240543

[3] Hobbs B., Nelson S., A nonlinear bilevel model for analysis of electric utility demand-side planning issues, Annals of Operations Research.1992; 34: 255-274. http://dx.doi.org/10.1007/BF02098182

[4] Marcotte P., Savard G., Bilevel programming: Applications, in: Encyclopedia of Optimization, Kluwer Academic Publishers, Dordrecht, 2001.

[5] Ben-Ayed O., Blair C., Computational difficulties of bilevel linear programming, Operations Research. 1990; 38: 556-560. http://dx.doi.org/10.1287/opre.38.3.556

[6] Blair C., The computational complexity of multi-level linear programs, Annals of Operations Research. 1992; 34: 13-19. http://dx.doi.org/10.1007/BF02098170

[7] Zadeh L., Fuzzy sets, Information and Control. 1965; 8: 338-353. http://dx.doi.org/10.1016/S0019-9958(65)90241-X

[8] Peidro D., Mula J., Jiménez M., Botella M., A fuzzy linear programming based approach for tactical supply chain planning in an uncertainty environment, European Journal of Operational Research. 2010; 205: 65-80. http://dx.doi.org/10.1016/j.ejor.2009.11.031

[9] Wu Z., Xu J., Nonconvex fuzzy mapping and the fuzzy pre-variational inequality, Fuzzy Sets and Systems. 2008; $159: 2090-2103$. http://dx.doi.org/10.1016/j.fss.2007.11.013

[10] Zimmermann H.J., Description and optimization in fuzzy systems, International Journal of General Systems. 1976 ; 2: $209-215$. http://dx.doi.org/10.1080/03081077608547470

[11] Zhang X., Huang G.H., Chan C., Liu Z., Lin Q., A fuzzy-robust stochastic multiobjective programming approach for petrolium waste management planing, Applied Mathematical Modelling. 2010; 34: 2778-2788. http://dx.doi.org/10.1016/j.apm.2009.12.012

[12] Liu S.T., Kao C., Network flow problems with fuzzy arc lengths, Transactions on Systems, Man, and Cybernetics. 2004; 34: 765-769. http://dx.doi.org/10.1109/TSMCB.2003.818560

[13] Yager R. A procedure for ordering fuzzy subsets of the unit interval, Information Sciences. 1981; 24: $143-161$. http://dx.doi.org/10.1016/0020-0255(81)90017-7

[14] Dempe S., Fanghänel D., Starostina T., Optimal toll charges: fuzzy optimization approach, in: F. Heyde, A. Löhne, C. Tammer (Eds.), Methods of Multicriteria Decision - Theory and Applications, Shaker Verlag, Aachen. 2009; 29-45.

[15] Dempe S., Starostina T., Optimal toll charges in a fuzzy flow problem, in: B. Reusch (Ed.). Computational Intelligence, Theory and Applications, Springer Verlag, Berlin. 2006; 405-413. http://dx.doi.org/10.1007/3-540-34783-6_41

[16] Bard J., Moore J., A branch and bound algorithm for the bilevel programming problem, SIAM Journal on Scientific and Statistical Computing. 1990; 11: 281-292. http://dx.doi.org/10.1137/0911017

[17] Ishizuka Y., Aiyoshi E. Double penalty method for bilevel optimization problems, Annals of Operations Research. 1992; 34: 73-88. http://dx.doi.org/10.1007/BF02098173

[18] Arora S., Gupta R., Interactive fuzzy goal programming approach for bilevel programming problem, European Journal of Operational Research. 2009; 194: 368-376. http://dx.doi.org/10.1016/j.ejor.2007.12.019

[19] Dubois D., Prade H., Operations on fuzzy numbers, International Journal of Systems Science. 1978; 9: 613-626. http://dx.doi.org/10.1080/00207727808941724

[20] Buckley J., Joint solution to fuzzy programming problems, Fuzzy Sets and Systems. 1995; 72: 215-220. http://dx.doi.org/10.1016/0165-0114(94)00353-9 
[21] Dempe S., Ruziyeva A., The Karush-Kuhn-Tucker optimality conditions in fuzzy optimization, The Annals of Fuzzy Sets, Fuzzy Logic and Fuzzy Systems. 2011; 1: 201-213.

[22] Panigrahi M., Panda G., Nanda S., Convex fuzzy mapping with differentiability and its application to fuzzy optimization, European Journal of Operational Research. 2008; 185: 47-62. http://dx.doi.org/10.1016/j.ejor.2006.12.053

[23] Wu H.C., The Karush-Kuhn-Tucker optimality conditions in an optimization problem with interval-valued objective function, European Journal of Operational Research 176 (2007) 46-59. http://dx.doi.org/10.1016/j.ejor.2005.09.007

[24] Dempe S., Ruziyeva A., On the calculation of a membership function for the solution of a fuzzy linear optimization problem, Fuzzy Sets and Systems. 2012; 188: 58-67. http://dx.doi.org/10.1016/j.fss.2011.07.014

[25] Dempe S., Lohse S., Optimale mautgebuehren - ein modell und ein optimalitätstest, Automatisierungstechnik. $2012 ; 4$ : 225 -232. http://dx.doi.org/10.1524/auto.2012.0990

[26] Dempe S., A simple algorithm for the linear bilevel programming problem, Optimization. 1987; 18: 373-385.

http://dx.doi.org/10.1080/02331938708843247 